\title{
Calling for an Integrated, Interdisciplinary and International Perspective on Mediated Communication Research
}

Mike Z Yao*

Department of Media and Communication, City University of Hong Kong, Kowloon, Hong Kong

New Year's Eve, Hong Kong. My small but close family was together. The television was on, but muted. On the stereo system, my favorite radio station in Los Angeles was streamed over the Internet. My wife and I were trying to set up a family videoconference with her parents in San Francisco. My 64-year old father, on his laptop computer, was browsing news about China on the Web and posting messages to his friends on Facebook while my mother was trying to figure out how to create a photo greeting card on her new iPad. To complete this family portrait, my 2-year old son was having an enthusiastic "conversation" with "Siri", the ever-so-patient voice recognizing and talking digital personal assistant on my smartphone. Of course, all of us were also talking to each other.

The scene above portrays the complexity of human communications today. It also highlights the growing importance of mediated communication in our daily lives. People around the world of all ages are spending more time communicating through media while information and communication technologies are becoming increasingly ubiquitous and omnipresent. Mediated communication takes place almost everywhere and at any time. It is replacing and displacing much of the face-to-face human interactions. Unlike just a decade ago, reading newspaper, viewing television, listening to radio, and chatting with friends are no longer separate activities nowadays. The lines between different communication contexts, such as interpersonal communication and mass communication, are blurred. The distinctions between various types of media content, such as news, advertising, and entertainment, are also disappearing.

The dramatic shifts in media technologies and people's communicative behavior in recent years undoubtedly introduced great challenges to communication researchers. There is a severe shortage of effective theoretical approaches to capture the vast, dynamic, and information-rich mediated space created by the Internet. As media devices and interfaces become increasingly mobile, interactive and integrated, many staple communication theories, particularly those hinged on particular modes of communication or focused on specific types of media content, are quickly becoming obsolete and irrelevant.

Communication research today might also be suffering from a lack of methodological tools. Conventional research methods in communication science are confronted with difficulties in the new mediated communication environment. For example, it is extremely difficult to find valid and representative samples in the cyberspace. The extent to which online and offline samples can be used to represent each other is also at question. Moreover, the interactive nature of new media technologies significantly hinders a researcher's ability to gain control in experimental designs. Existing sampling and coding techniques of content analyses are simply incapable of handling the astronomical amount of digital information.

Although communication researchers today face conceptual, theoretical, as well as methodological barriers, I believe the field as a whole is at a historic moment in moving towards a more central position in social and behavioral sciences. The unprecedented development and adoption of interactive information and communication technologies in the past few years have attracted much attention from scholars in all scientific disciplines. A quick review of recent publications in cognitive and information sciences, computer engineering, and even physics would show that researchers in many fields are conducting interesting studies to examine the social and behavioral phenomena we have been studying for years. While there is a flux of related research being produced in various social and information science disciplines recently, there is very little interdisciplinary collaboration. The same communication processes are often studied by researchers in different fields, from different perspectives, and with different methods.

I believe that communication scientists, armed with the knowledge from nearly a century of systematic study on media and their social and psychological impacts, are best equipped to integrate various theoretical perspectives from different fields. However, such an endeavor cannot be superficial and variable analytic. Meaningful theory integration must begin with rigorous and thorough concept explication. The integration should be both vertical and horizontal. Vertically, theories developed for various levels and contexts of communication, such as interpersonal communication and mass communication ought to be considered together. Horizontally, theoretical and methodological knowledge generated in non-social science disciplines, such as information and cognitive sciences and computer engineering, should also be critically evaluated and incorporated.

In addition to taking an integrated and interdisciplinary approach, the study of mediated communication should be more international. As the scenario at the beginning of this editorial shows, the idea of globalization is not only a fancy word used by politicians and scholars; it has deep impact on the communicative and information seeking behaviors of average people across the world. Furthermore, contemporary communication research has been too America centric for too long. Many dominant communication theories we rely on were developed from empirical works done in the North America. Even though these theories have been well tested and retested by American

*Corresponding author: Mike Z Yao, Department of Media and Communication, City University of Hong Kong, Kowloon, Hong Kong, Tel: 3442 8611; Fax: 3442 0228; E-mail: mike.yao@cityu.edu.hk

Received January 16, 2012; Accepted January 16, 2012; Published January 18 2012

Citation: Yao MZ (2012) Calling for an Integrated, Interdisciplinary and International Perspective on Mediated Communication Research. J Mass Communicat Journalism 2:e111. doi:10.4172/2165-7912.1000e111

Copyright: (c) 2012 Yao MZ. This is an open-access article distributed under the terms of the Creative Commons Attribution License, which permits unrestricted use, distribution, and reproduction in any medium, provided the original author and source are credited. 
Citation: Yao MZ (2012) Calling for an Integrated, Interdisciplinary and International Perspective on Mediated Communication Research. J Mass Communicat Journalism 2:e111. doi:10.4172/2165-7912.1000e111

Page 2 of 2

researchers, rarely were they systematically examined in other cultural and geopolitical contexts. The study of mediated communication in a globalized world ought to consider the applicability of our theories in difficult cultural and geopolitical settings.

In summary, I firmly believe that our field is at the cusp of a historical change. In the era when product development cycles of media technologies are shorter than the publication cycles of many academic journals, researchers cannot afford to mindlessly apply existing theories to examine novel communication phenomena without critical evaluation. Such studies will be quickly obsolete. More important than ever, we need to think about fundamental issues of human communication at the conceptual and meta-theoretical levels. Mediated human communication must be carefully explicated and studied from an integrated, interdisciplinary, and international perspective. 